\title{
Polarization - interference mapping of the distributions of the parameters of the Stokes vector of the object field of a biological optically anisotropic layer
}

\author{
A. Dubolazov ${ }^{1}$, V. Ushenko ${ }^{1}$, O. Litvinenko ${ }^{2}$, V. Bachinskiy ${ }^{2}$, A. Petrushak $^{2}$, \\ A. Karachevtsev ${ }^{1}$, M.L. Kovalchuk ${ }^{1}$ \\ ${ }^{1}$ Chernivtsi National University, 2 Kotsiubynskyi Str., Chernivtsi, Ukraine, 58012 \\ ${ }^{2}$ Bukovinian State Medical University, 3 Theatral Sq., Chernivtsi, Ukraine, 58000
}

\begin{abstract}
The materials of experimental testing of the Stokes-polarimetry method using a reference laser wave are presented. The results of layer-by-layer measurement of coordinate distributions of the magnitude of the ellipticity of the polarization of laser radiation converted by polycrystalline films of biological fluids are presented. In the framework of the statistical and cross-correlation approaches, the values and ranges of changes in the statistical and correlation moments of the 1st to 4th orders of magnitude characterizing the distribution of the ellipticity of the polarization of laser radiation converted by polycrystalline networks in different phase sections are determined.
\end{abstract}

Keywords: interference, polarization, Stokes vector, anisotropy, diagnostic.

\section{INTRODUCTION}

Optical sheme and measurement procedure

The Stokes-correlometric mapping scheme is shown in fig. $1^{1-4}$.

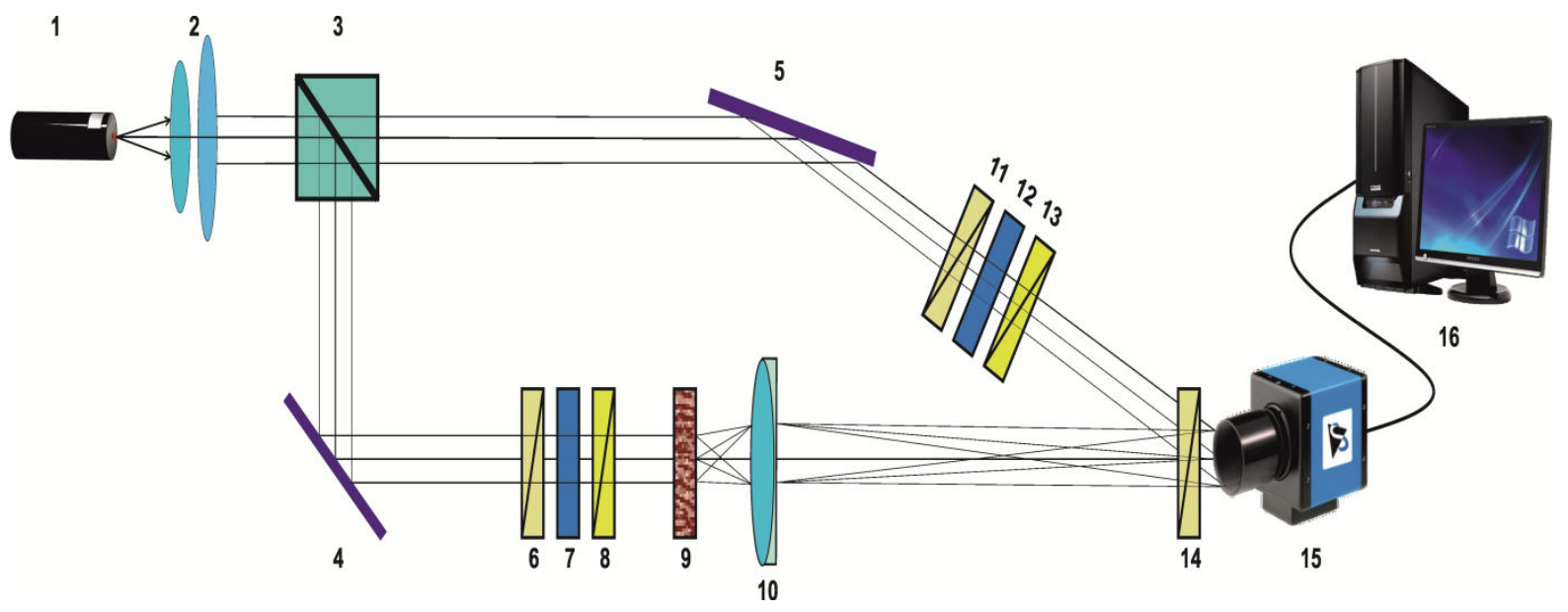

Figure 1. The optical scheme of polarization-interference mapping of the parameters of the Stokes vector of optically anisotropic biological layers.

Fourteenth International Conference on Correlation Optics, edited by

Oleg V. Angelsky, Proc. of SPIE Vol. 11369, 113691N · (c) 2020 SPIE

CCC code: $0277-786 \mathrm{X} / 20 / \$ 21 \cdot$ doi: $10.1117 / 12.2553953$ 
A parallel $\left(\varnothing=2 \times 10^{3} \mu \mathrm{m}\right)$ beam of He-Ne $(\lambda=0,6328 \mu \mathrm{m})$ laser 1 , formed using a collimator 2 , using $50 \%$ beam splitter 3 , is divided into "irradiating" and "reference".

The "irradiating" using a rotating mirror 4 is sent through a polarizing filter 6 - 8 in the direction of the sample of the biological layer 9. A polarizing-inhomogeneous image of the object 9 with the lens 10 is projected into the plane of the digital camera 14. The "reference" beam is directed by the mirror 5 through the polarization filter $11-13$ into the plane of the polarization-inhomogeneous image of the object 9. As a result, an interference pattern is formed, the coordinate intensity distribution of which is recorded by the digital camera 14 . The method of polarization-correlation determination of the set of parameters of the Stokes vector consists in the following set of actions:

- The simultaneous formation in the "irradiating" and "reference" parallel laser beams of one of these polarization states (for example

$$
\left.\left(0^{0}-0^{0}\right) ; \quad\left(90^{0}-90^{0}\right) ; \quad\left(45^{0}-45^{0}\right) ; \quad\left(135^{0}-135^{0}\right) ;(\otimes-\otimes) ; \quad(\oplus-\oplus)\right) .
$$

- For each of these polarization states, registration of each partial interference pattern is experimentally carried out through the polarizer-analyzer 14 with the orientation of the transmission plane at angles $\Theta=0^{0} ; \quad \Theta=90^{0}$.

- Recovery for each partial polarization-interference distribution in the aggregate of phase cross $\varphi=c o n s t$ sections using the integrated diffraction transformation of coordinate distributions of complex amplitudes $\left\{E_{x}(\varphi, r) ; \quad E_{y}(\varphi, r)\right\}^{6-12}$ in the plane of the microscopic image of the biological layer.

- Calculation in each phase plane $\left(\varphi_{k}=k \Delta \varphi, k=0 ; 1 ; 2 ; \ldots\right)$ of coordinate distributions $(x, y)$ of a set of Stokes vector parameters $S_{i=1 ; 2 ; 3 ;}(\varphi, x, y)$ and polarization parameters $\alpha(\varphi, x, y)$ and $\beta(\varphi, x, y)$ according to the following algorithms

$$
\begin{gathered}
S_{1}\left(\varphi_{k}, x, y\right)=\left(\left|E_{x}\right|^{2}+\left|E_{y}\right|^{2}\right)\left(\varphi_{k}, x, y\right) ; \\
S_{2}\left(\varphi_{k}, x, y\right)=\left(\left|E_{x}\right|^{2}-\left|E_{y}\right|^{2}\right)\left(\varphi_{k}, x, y\right) ; \\
S_{3}\left(\varphi_{k}, x, y\right)=2 \operatorname{Re}\left|E_{x} E_{y}^{*}\right|\left(\varphi_{k}, x, y\right) ; \\
S_{4}\left(\varphi_{k}, x, y\right)=2 \operatorname{Im}\left|E_{x} E_{y}^{*}\right|\left(\varphi_{k}, x, y\right) . \\
\alpha\left(\varphi_{k}, x, y\right)=0,5 \operatorname{arctg}\left[\frac{S_{3}\left(\varphi_{k}, x, y\right)}{S_{2}\left(\varphi_{k}, x, y\right)}\right] ; \\
\beta\left(\varphi_{k}, x, y\right)=0,5 \operatorname{arctg}\left[\frac{S_{4}\left(\varphi_{k}, x, y\right)}{S_{1}\left(\varphi_{k}, x, y\right)}\right] .
\end{gathered}
$$

\section{THREE-DIMENSIONAL MAPS OF ELLIPTICITY OF POLARIZATION OF DENDRITIC POLYCRYSTALLINE NETWORKS OF LIQUOR FILMS}

As test samples, we used optically thin films of liquor with optical thicknesses $\tau=0,089 \div 0,096$, within which the condition for a single scattering of laser radiation by a dendritic network of needle crystals is realized.

The ellipticity of polarization was chosen as the main information parameter (without reducing the completeness of the analysis), the value of which is integrally determined by both the birefringence of biochemical crystals and the directions of the optical axes ${ }^{12-22}$.

The "reference" phase planes in the process of 3D reproduction of distributions of polarization ellipticity values were $\varphi=0,4 \mathrm{rad} ; 0,8 \mathrm{rad} ; 1,2 \mathrm{rad}$. The results of layer-by-layer reproduction of polarization ellipticity maps are shown in a series of dependencies in fig. 1, fig. 4 and fig. 7. 
Fig. 2, fig. 5 and fig. 8 illustrates the topographic structure $(50$ pix $\times 50$ pix $)$ of the map of the ellipticity of the polarization of laser radiation in the thickness of a polycrystalline liquor film in various phase sections.

The data of cross-correlation analysis of layer-by-layer distributions of polarization ellipticity values are presented as a series of autocorrelation functions in fig. 3, fig. 6 and fig. 9 .

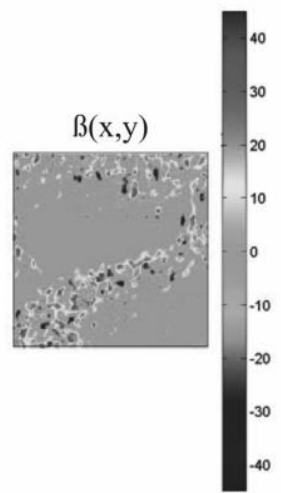

(1)

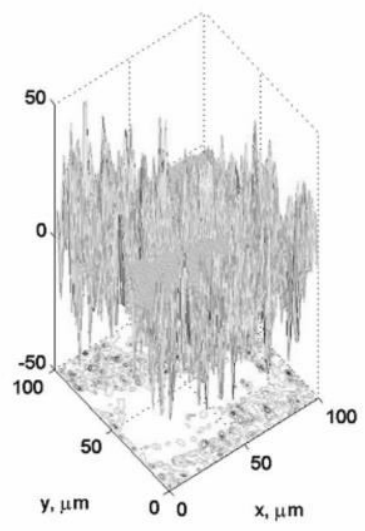

(2)

Figure 2. Coordinate (fragment (1)) and volume (fragment (2)) structure of the map of the ellipticity of polarization of the polycrystalline cerebrospinal fluid film in the phase section $\varphi=0,4$.

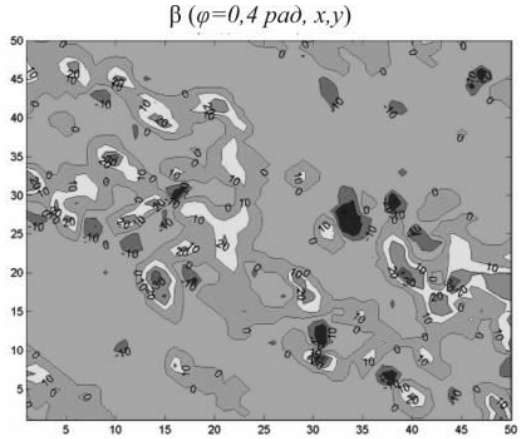

Figure 3. Topographic structure $(50$ pix $\times 50$ pix $)$ of the polarization ellipticity map of the polycrystalline liqour film in the phase section. Explanation in the text.

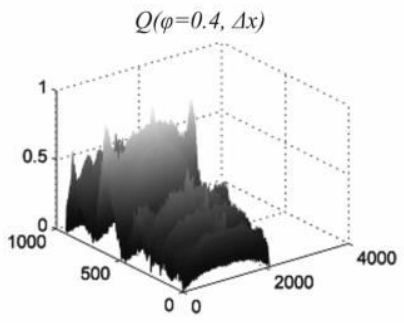

(1)

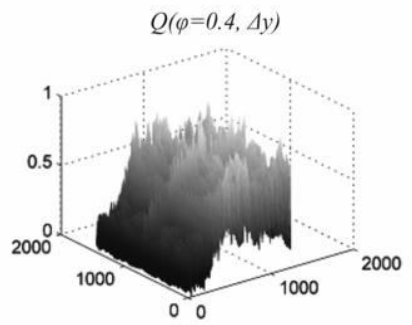

(2)

Figure 4. Cross-correlation distribution functions of the polarization ellipticity of the polycrystalline liquor film in the phase section $\varphi=0,4$. Explanations in the text. 


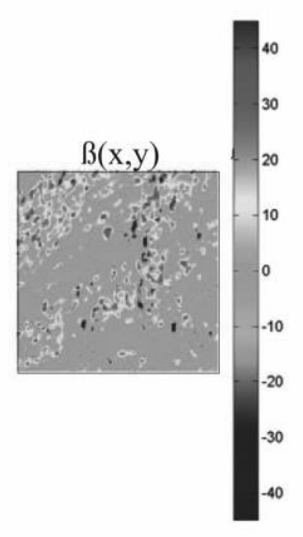

(1)

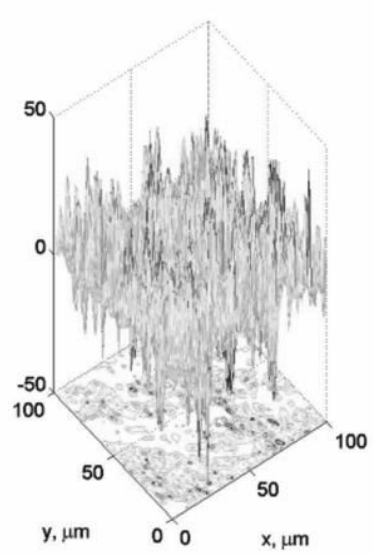

(2)

Figure 5. Coordinate (fragment (1)) and volumetric (fragment (2)) structure of the polarization ellipticity map of the polycrystalline liquor film in the phase section $\varphi=0,8$.

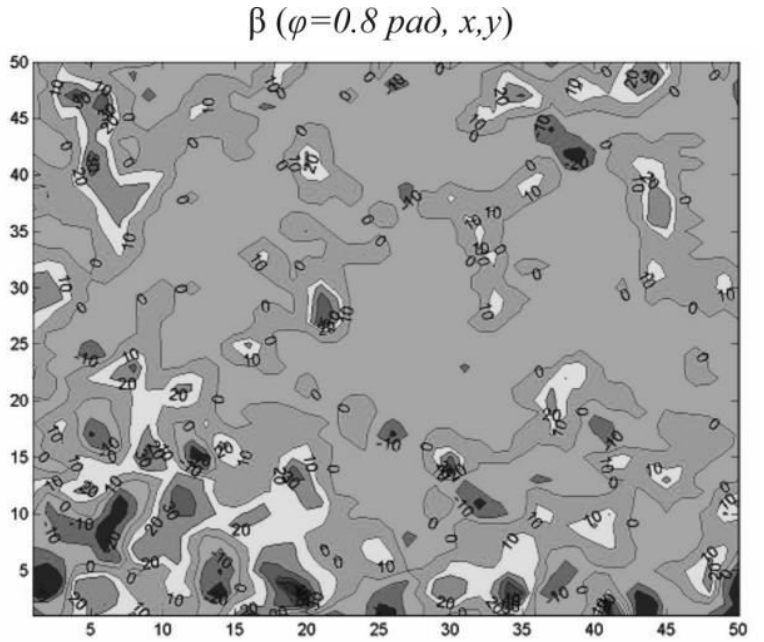

Figure 6 . Topographic structure $(50$ pix $\times 50$ pix $)$ of the polarization ellipticity map of the polycrystalline liquor film in the phase section $\varphi=0,8$.

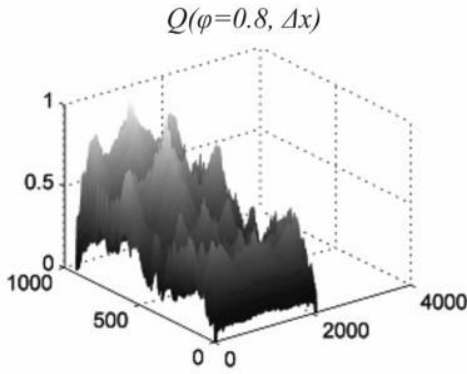

(1)

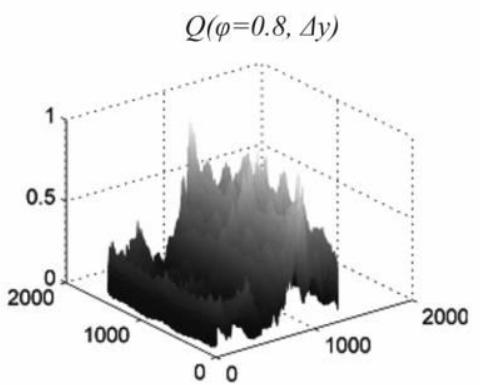

(2)

Fig. 7. Cross-correlation distribution functions of the polarization ellipticity of the polycrystalline liquor film in the phase section $\varphi=0,8$. 


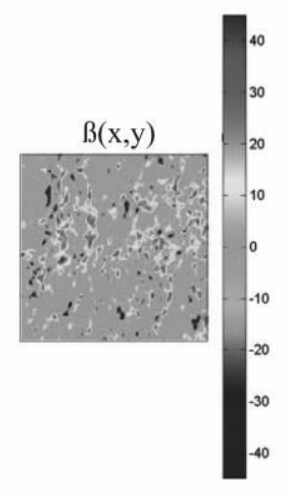

(1)

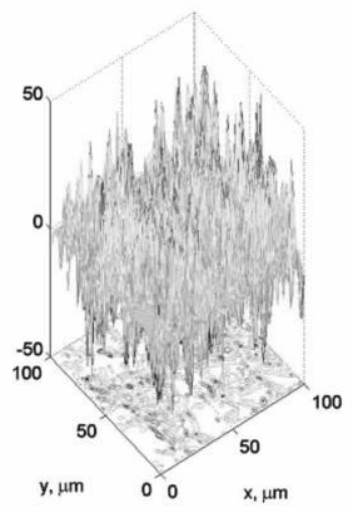

(2)

Fig. 8. Coordinate (fragment (1)) and volumetric (fragment (2)) structure of the polarization ellipticity map of the polycrystalline liquor film in the phase section $\varphi=1,2$.

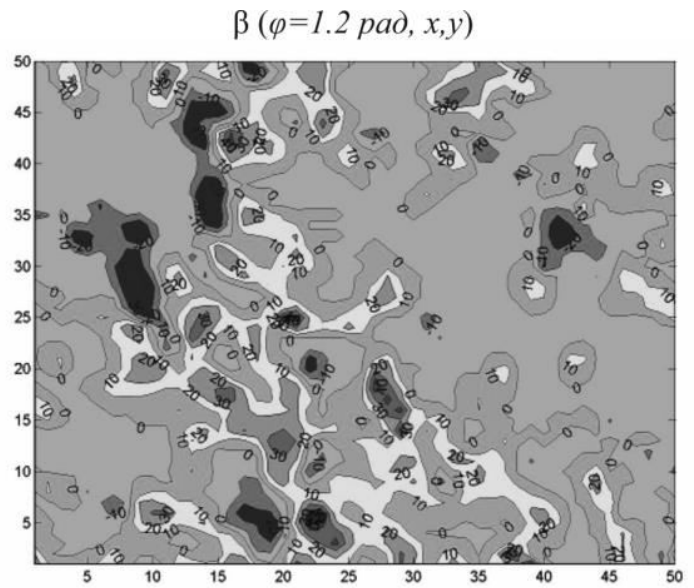

Fig. 9. Topographic structure $(50$ pix $\times 50$ pix $)$ of the polarization ellipticity map of a polycrystalline liquor film in phase section $\varphi=1,2$.

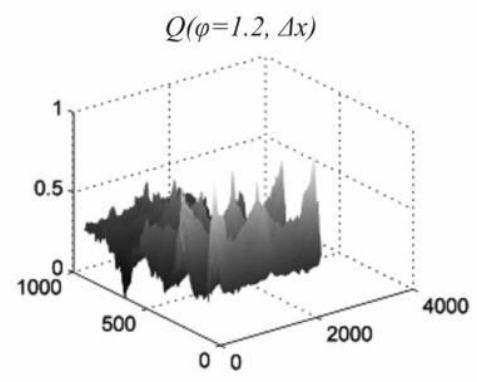

(1)

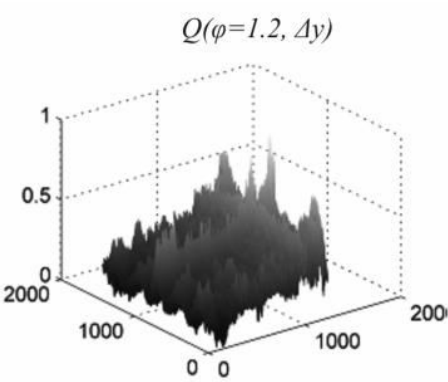

(2)

Fig. 10. Cross-correlation distribution functions of the polarization ellipticity of the polycrystalline liquor film in the phase section $=\varphi 1,2$. 
An analysis of the data obtained by polarization mapping in various phase sections of the laser radiation field transformed by a polycrystalline network of dendritic crystals of the liquor film (fig. 1 - fig. 9) revealed:

1. The main polarimetric manifestations of the polycrystalline structure of the optically anisotropic liquor layer is the formation of distributions of the ellipticity of polarization (fig. 1, fig. 2, fig. 4, fig. 5, fig. 7, fig. 8, fragments (1), (2) ).

2. For each of the coordinate distributions $\beta\left(\varphi_{i}, x, y\right)$, within the partial phase cross sections $\varphi_{i}=0,4 \mathrm{rad} ; 0,8 \mathrm{rad} ; 1,2 \mathrm{rad}$, an individual statistical inherent (probability and range of variation of the polarization ellipticity - fig. 1, fig. 4, fig. 7, fragments (2)) and topographic (features of the coordinate distribution of values $\beta$ ) structure - fig. 2 , fig. 5 , fig. 8 .

3. The topographic structure of the polarization ellipticity maps is a collection of local domains $\beta(\Delta x, \Delta y) \approx$ const that are randomly located in the plane of phase sections $\varphi_{i}$ of the laser radiation field transformed by a polycrystalline liquor film - fig. 2, fig. 5 , fig. 8.

4. With increasing $(\uparrow)$ of the phase cross section $(\varphi \uparrow)$ of the polarization-inhomogeneous field $(\beta(x, y))$, the range of changes in the local values of the polarization ellipticity $(\Delta \beta \uparrow)$ and the values themselves $\beta$ increase - fig. 2 , fig. 5 , fig. 8 respectively.

5. The autocorrelation functions $Q\left(\varphi_{i}, \Delta x\right)$ and $Q\left(\varphi_{i}, \Delta y\right)$, characterizing the degree of coordination of polarization maps $\beta\left(\varphi_{i}, x, y\right)$ in orthogonal directions ( $O x$ and $O y$ ), are complex descending dependences with fluctuations $\left(\tilde{Q}\left(\varphi_{i}, \Delta x\right)\right.$ and $\left.\tilde{Q}\left(\varphi_{i}, \Delta y\right)\right)$ of the eigenvalues of the dependences - fig. 3, fig. 6, fig. 9.

6. With increasing $(\uparrow)$ of the phase cross section $(\varphi \uparrow)$ of the polarization-inhomogeneous field $(\beta(x, y))$, the halfwidth of the dependences of the cross-correlation functions $Q\left(\varphi_{i}, \Delta x\right)$ and $Q\left(\varphi_{i}, \Delta y\right)$ decreases, as well as the amplitude of the fluctuations $\tilde{Q}\left(\varphi_{i}, \Delta x\right)$ and $\tilde{Q}\left(\varphi_{i}, \Delta y\right)$.

Let us analyze the results obtained from a physical point of view within the statistical and correlation approaches.

Statistical analysis. A polycrystalline dendritic network is formed by planar needle-shaped linearly birefringent $(\delta)$ albumin crystals with randomly located directions $(\rho)$ of optical axes located in the plane of the liquor film.

Thus, the optical-anisotropic properties of the liquor film are determined by the distributions of phase $\Phi_{\delta}$ and orientation $\Phi_{\rho}$ parameters. When polarized laser radiation passes through a local crystal with random parameters $\rho_{i}, \delta_{i}$, a random value of polarization ellipticity $\beta_{i}$ is formed. As a result, in a certain partial plane, a two-dimensional distribution of random ellipticity values $\Phi_{\beta}$ is formed. A quantitative assessment of such a distribution is a set of statistical moments of the 1 st - 4 th orders $Z_{i=1 ; 2 ; 3 ; 4}(\beta)$.

In our situation, by varying the increasing value of the phase cross section, it is possible to sequentially detect $\Phi_{\beta}\left(\varphi_{i}\right)$ the distributions in various partial planes of the polycrystalline liquor film. It should be noted that for small values $\varphi_{i}$ (in our case $\varphi=0,4 \mathrm{pad}$ ), the most probable are coordinate-localized separate acts of interaction of laser radiation with individual crystals. Due to this, the distribution map of the polarization ellipticity $\beta(\mathrm{x}, \mathrm{y})$ is a collection of local polarization domains $\beta(\Delta x, \Delta y) \approx$ const with a small range of variation of the ellipticity value $\left(-20^{0} \leq \beta \leq 20^{0}\right)$ against the background of the most probable linearly polarized radiation $\left(\beta=0^{0}\right)$, which corresponds to optically isotropic sections of the liquor film - fig. 2. Therefore, such a distribution $\Phi_{\beta}\left(\varphi_{i}=0,4 \mathrm{pad}\right)$ is generally characterized by small values of statistical moments of the 1st (average) and 2nd (dispersion) orders and, conversely, by a significant level of asymmetry $\left(\mathrm{Z}_{3}(\beta)\right)$ and excess $\left(\mathrm{Z}_{4}(\beta)\right)$. 
For large values of the phase cross section (in our case $\varphi=0,8 \mathrm{rad} ; 1,2 \mathrm{rad}$ ), the probability of interaction with several optically anisotropic crystalline formations of the dendritic network increases. As a result, the quantity, area, size, and range of ellipticity changes within the polarization domains - fig. 5 and fig. 8. Quantitatively, this is manifested in opposing trends in the magnitude of statistical moments $-Z_{1}(\beta)$ and $Z_{2}(\beta)$ - grow; statistical moments of higher orders, on the contrary, decrease.

Correlation analysis. A comparative analysis of the coordinate structure of maps of polarization ellipticity $\beta\left(\varphi_{i}, x, y\right)$ in various phase sections $\varphi_{i}$ revealed the following general features. First, the coordinate heterogeneity of the topographic structure of polarization domains. Secondly, the location of such polarization sites $(\beta(\Delta x, \Delta y) \approx c o n s t)$ is repeated with a certain frequency - fig. 2, fig. 5 , fig. 8 .

The first factor is manifested in a fairly rapid drop in the eigenvalues of autocorrelation functions $Q$, which were determined in two mutually perpendicular directions. The second - leads to the formation of quasiperiodic fluctuations ( $\tilde{Q}\left(\varphi_{i}, \Delta x\right)$ and $\left.\tilde{Q}\left(\varphi_{i}, \Delta y\right)\right)$ of the value of cross-correlation functions $Q\left(\varphi_{i}, \Delta x\right)$ and $Q\left(\varphi_{i}, \Delta y\right)$.

An increase in the phase cross section (in our case $\varphi=0,8 \mathrm{rad} ; 1,2 \mathrm{rad}$ ) leads to a decrease in the half-width of crosscorrelation functions $Q\left(\varphi_{i}, \Delta x\right)$ and $Q\left(\varphi_{i}, \Delta y\right)$ an increase in the sharpness of their peak, as well as a decrease in the amplitude of their fluctuations $\tilde{Q}\left(\varphi_{i}, \Delta x\right), \tilde{Q}\left(\varphi_{i}, \Delta y\right)$. The reason for this is the increase in the coordinate inhomogeneity of the polarization-inhomogeneous laser field in these planes. In particular, the number of local polarization domains is increasing, within the area of which the magnitude and range of the ellipticity change also increase - Fig. 5 and fig. 8 due to an increase in the probability of interaction of laser radiation with several optically anisotropic crystalline formations of the dendritic network. From a physical point of view, such a process indicates the formation of a more polarizationinhomogeneous field of laser radiation transformed by an ensemble of crystals of a dendritic network of a liquor film. Quantitatively, this is manifested in opposite trends in the magnitude of correlation moments - the average value ( $\left.K_{1}(\beta)\right)$ and half-width $\left(K_{2}(\beta)\right)$ of cross-correlation functions $Q\left(\varphi_{i}, \Delta x\right)$ and $Q\left(\varphi_{i}, \Delta y\right)$ polarization ellipticity maps $\beta\left(\varphi_{i}, x, y\right)$ decrease. Correlation moments of higher orders $\left(K_{3 ; 4}(\beta)\right.$ - asymmetry and excess of cross-correlation ) increase, on the contrary, with increasing a value of the phase section of the 3D distribution of the polarization ellipticity of the polycrystalline liquor film. The values of statistical $Z_{i=1 ; 2 ; 3 ; 4}$ and correlation $K_{j=1 ; 2 ; 3 ; 4}$ moments of the 1st to 4 th orders of magnitude that characterize the coordinate distributions and cross-correlation functions of the magnitude of the polarization ellipticity $\beta$ of the three-dimensional microscopic image of the polycrystalline dendritic network of the liquor film in various phase sections $\varphi$ are shown in table 1 .

Table 1. Statistical and correlation moments that characterize the distribution of the magnitude of the polarization elliptic $\beta$ of the microscopic image of the polycrystalline liquor film in various phase sections $\varphi$

\begin{tabular}{|c|c|c|c|c|c|}
\hline \multicolumn{3}{|c|}{$Z_{i}(\beta)$} & \multicolumn{3}{|c|}{$K_{j}(\beta)$} \\
\hline \multirow[t]{4}{*}{$\varphi=0,4$} & $Z_{1}$ & 0,09 & \multirow[t]{4}{*}{$\varphi=0,4$} & $K_{1}$ & 0,19 \\
\hline & $Z_{2}$ & 0,11 & & $K_{2}$ & 0,12 \\
\hline & $Z_{3}$ & 1,18 & & $K_{3}$ & 0,47 \\
\hline & $Z_{4}$ & 1,57 & & $K_{4}$ & 0,65 \\
\hline \multirow[t]{4}{*}{$\varphi=0,8$} & $Z_{1}$ & 0,13 & \multirow[t]{4}{*}{$\varphi=0,8$} & $K_{1}$ & 0,12 \\
\hline & $Z_{2}$ & 0,15 & & $K_{2}$ & 0,08 \\
\hline & $Z_{3}$ & 0,87 & & $K_{3}$ & 1,03 \\
\hline & $Z_{4}$ & 1,09 & & $K_{4}$ & 1,49 \\
\hline
\end{tabular}




\begin{tabular}{|c|c|c|c|c|c|}
\hline \multirow[t]{4}{*}{$\varphi=1,2$} & $Z_{1}$ & 0,19 & \multirow[t]{4}{*}{$\varphi=1,2$} & $K_{1}$ & 0,07 \\
\hline & $Z_{2}$ & 0,21 & & $K_{2}$ & 0,05 \\
\hline & $Z_{3}$ & 0,53 & & $K_{3}$ & 1,28 \\
\hline & $Z_{4}$ & 0,48 & & $K_{4}$ & 1,87 \\
\hline
\end{tabular}

A comparative analysis ${ }^{23-28}$ of the change in the magnitude of the 1 st and 4 th order statistical and correlation moments in different phase sections of the 3D distribution of the polarization ellipticity of the object field of the polycrystalline liquor film revealed significant differences between them:

- $\Delta Z_{1}(\varphi)=2,1 ; \Delta Z_{2}(\varphi)=1,9 ; \Delta Z_{3}(\varphi)=2,3 ; \Delta Z_{4}(\varphi)=2,3$

- $\Delta K_{1}(\varphi)=2,3 ; \Delta K_{2}(\varphi)=2,4 ; \Delta K_{3}(\varphi)=2,7 ; \Delta K_{4}(\varphi)=2,9$.

\section{CONCLUSIONS}

The most sensitive to changes in the parameters of linear birefringence $(\rho, \delta)$ of the polycrystalline network of dendritic crystals in the volume of the liquor film were statistical ( $Z_{i=3 ; 4}$ - differences for different phase cross sections are 2.3 times) and correlation ( $K_{i=3 ; 4}$ - differences for different phase cross sections are 2.7 - 2, 9 times) moments of higher orders characterizing the distribution of the ellipticity $\beta(\varphi, x, y)$ of the polarization-inhomogeneous object field of laser radiation.

\section{REFERENCES}

[1]. Wang X. Propagation of polarized light in birefringent turbid media: a Monte Carlo study / X. Wang, L.-H. Wang // J. Biomed. Opt. - 2002. - Vol. 7. - P. 279-290.

[2]. Tuchin V. V. Handbook of optical biomedical diagnostics / V. V. Tuchin. - Bellingham : SPIE Press, 2002. - 1110 p.

[3]. Yao G. Two-dimensional depth-resolved Mueller matrix characterization of biological tissue by optical coherence tomography / G. Yao, L. V. Wang // Opt. Lett. - 1999. - V. 24. - P. 537-539.

[4]. Tower T. T. Alignment Maps of Tissues: I. Microscopic Elliptical Polarimetry / T. T. Tower, R. T. Tranquillo // Biophys. J. - 2001. - Vol. 81. - P. 2954-2963.

[5]. Lu S. Interpretation of Mueller matrices based on polar decomposition / S. Lu, R. A. Chipman // J. Opt. Soc. Am. A. 1996. - Vol. 13. - P.1106-1113.

[6]. Ghosh Nirmalya. Techniques for fast and sensitive measurements of two-dimensional birefringence distributions / Nirmalya Ghosh, I. Alex Vitkin // Journal of Biomedical Optics. - 2011. - № 16(11). - P. 110801.

[7]. V. V. Tuchin, L. Wang, and D. A . Zimnyakov, Optical Polarization in Biomedical Applications, New York, USA (2006).

[8]. Angelsky, O.V., Hanson, S.G., Maksimyak, P.P., Maksimyak, A.P., Zenkova, C.Yu., Polyanskii, P.V., Ivanskyi, D.I., "Influence of evanescent wave on birefringent microplates," (2017) Optics Express, 25 (3), pp. 2299-2311.

[9]. Angelsky, O.V., Ushenko, Y.A., Dubolazov, A.V., Telenha, O.Yu., "The interconnection between the coordinate distribution of mueller-matrixes images characteristic values of biological liquid crystals net and the pathological changes of human tissues,"(2010) Advances in Optical Technologies, art. no. 130659.

[10]. Bekshaev, A.Ya., Angelsky, O.V., Sviridova, S.V., Zenkova, C.Yu., "Mechanical action of inhomogeneously polarized optical fields and detection of the internal energy flows," (2011) Advances in Optical Technologies, art. no. 723901.

[11]. Angelsky, O.V., Maksimyak, P.P., Perun, T.O., "Optical correlation method for measuring spatial complexity in optical fields," (1993) Optics Letters, 18 (2), pp. 90-92.

[12]. Angelsky, O.V., Ushenko, A.G., Ushenko, Y.A., Pishak, V.P., "Statistical and fractal structure of biological tissue mueller matrix images," (2007) Optical Correlation Techniques and Applications, pp. 213-265. 
[13]. Angelsky, O.V., Ushenko, A.G., Pishak, V.P., Burkovets, D.N., Yermolenko, S.B., Pishak, O.V., Ushenko, Yu.A., "Coherent introscopy of phase-inhomogeneous surfaces and layers," (2000) Proceedings of SPIE - The International Society for Optical Engineering, 4016, pp. 413-418.

[14]. Angelsky, O.V., "Optical correlation techniques and applications,"(2007) Optical Correlation Techniques and Applications, pp. 1-270.

[15]. Angelsky, O.V., Maksimyak, P.P., “Optical diagnostics of slightly rough surfaces,” (1992) Applied Optics, 31 (1), pp. 140-143.

[16]. Angelsky, O.V., Maksimyak, P.P., "Polarization-interference measurement of phase-inhomogeneous objects," (1992) Applied Optics, 31 (22), pp. 4417-4419.

[17]. Angelsky, O. V., Bekshaev, A. Ya., Maksimyak, P. P., Maksimyak, A. P., Hanson, Steen Grüner, "Low-temperature laser-stimulated controllable generation of micro-bubbles in a water suspension of absorptive colloid particles," Optics Express (2018), Vol. 26, No. 11. pp. 13995-14009.

[18]. Ushenko, Yu.A., Tomka, Yu.Ya., Dubolazov, A.V., Telen'ga, O.Yu. Diagnostics of optical anisotropy changes in biological tissues using Müller matrix (2011) Quantum Electronics, 41 (3), pp. 273-277.

[19]. Ushenko, Yu.A., Tomka, Yu.Ya., Dubolazov, A.V. Laser diagnostics of anisotropy in birefringent networks of biological tissues in different physiological conditions (2011) Quantum Electronics, 41 (2), pp. 170-175.

[20]. Ushenko, Y.A., Dubolazov, O.V., Karachevtsev, A.O. Statistical structure of skin derma Mueller matrix images in the process of cancer changes (2011) Optical Memory and Neural Networks (Information Optics), 20 (2), pp. 145-154.

[21]. Ushenko, V.A., Dubolazov, A.V. Correlation and self similarity structure of polycrystalline network biological layers mueller matrices images (2013) Proceedings of SPIE - The International Society for Optical Engineering, 8856, 88562D.

[22]. Ushenko, A.G., Dubolazov, A.V., Ushenko, V.A., Novakovskaya, O.Y. Statistical analysis of polarizationinhomogeneous Fourier spectra of laser radiation scattered by human skin in the tasks of differentiation of benign and malignant formations (2016) Journal of Biomedical Optics, 21 (7), 071110.

[23]. Ushenko, Y.A., Dubolazov, A.V., Angelsky, A.P., Sidor, M.I., Bodnar, G.B., Koval, G., Zabolotna, N.I., Smolarz, A., Junisbekov, M.S. Laser polarization fluorescence of the networks of optically anisotropic biological crystals (2013) Proceedings of SPIE - The International Society for Optical Engineering, 8698, 869809.

[24]. Angelsky, O. V., Pishak, V. P., Ushenko, A. G., \& Ushenko, Y. A., " Statistical and fractal structure of biological tissue Mueller matrix images," Optical Correlation: Techniques and Applications, 213 (2007).

[25]. Ushenko, A. G., Ermolenko, S. B., Burkovets, D. N., \& Ushenko, Y. A., "Polarization microstructure of laser radiation scattered by optically active biotissues," Optics and Spectroscopy 87, 434-438 (1999).

[26]. Angelsky, O. V., Ushenko, A. G., \& Ushenko, Y. A., "Polarization reconstruction of orientation structure of biological tissues birefringent architectonic nets by using their Mueller-matrix speckle-images," Journal of Holography and Speckle 2(2), $72-79$ (2005).

[27]. Angelsky, O. V., Yermolenko, S. B., Prydij, O., Ushenko, A. G., Ushenko, Y. A., \& Ushenko, Y. G., "Polarizationinterference structure of speckle fields of the rough skin surface," Journal of Holography and Speckle 3(1), 27-34 (2006).

[28]. V. A. Ushenko, N. I. Zabolotna, S. V. Pavlov, D. M. Burcovets, O. Yu. Novakovska, Olexander V. Dubolazov, "Mueller-matrices polarization selection of two-dimensional linear and circular birefringence images," Proc. SPIE 9066, Eleventh International Conference on Correlation Optics, 90661X (17 December 2013). 